\title{
Review
}

Hans U. Fuchs*

\section{A Direct Entropic Approach to Uniform and Spatially Continuous Dynamical Models of Thermoelectric Devices}

\begin{abstract}
If we accept temperature and entropy as primitive quantities, we can construct a direct approach to a dynamical thermal theory of spatially continuous and uniform processes. The theory of uniform models serves as a simple entry point for learners of modern thermodynamics. Such models can be applied fruitfully to an understanding of (the dynamics of) thermoelectric processes and devices. Entropy, temperature, charge, and voltage allow us to describe the role of energy concisely, and constitutive quantities can be given their natural entropic interpretation. In this paper, aggregate dynamical models of a Peltier device will be created and simulations will be compared to non-steady-state experimental data. Such overall models give us a simple image of the transport of charge and transport, production, and storage of entropy and can be easily extended to the spatially continuous case. Process diagrams for a uniform model can be used to visualize these processes and the role of energy. Device efficiency can be easily read from the model. Apart from external parameters such as load resistances or temperature differences, it depends upon three parameters of the device: internal electric resistance, entropy conductance, and Seebeck coefficient. The Second Law efficiency of a generator suggests how to define the figure of merit $(z T)$ of the thermoelectric material. Distinction between ideal and dissipative processes and the rates at which energy is made available or used allows us to construct a simple argument for the equality of the Seebeck and Peltier coefficients.
\end{abstract}

Keywords: thermoelectricity, entropy, dynamic thermal models, reciprocity relations, Second Law efficiency

DOI 10.1515/ehs-2014-0011

*Corresponding author: Hans U. Fuchs, Institute of Applied Mathematics and Physics, Zurich University of Applied Sciences at Winterthur, 8400 Winterthur, Switzerland,

E-mail: hans.fuchs@zhaw.ch

\section{Introduction}

The goal of this paper is to provide a macroscopic approach to thermoelectric processes suitable for a first course in physics, physical chemistry, or thermodynamics. This approach starts with phenomenology and uses an interpretation of temperature as the thermal potential and entropy as the fundamental property that is transported in thermal process (what in lay terms would be called "heat"). The resulting theory of the creation, flow, and balance of entropy provides the foundation of a truly dynamical theory of heat that unites thermodynamics and heat transfer into a single subject. Conceptualizing entropy in this manner makes it clear that it corresponds to Carnot's caloric if we allow for caloric to be created in irreversible processes. Such an interpretation leads to visual metaphors that serve as a guide to model building - as we will show by applying it to thermoelectricity.

The presentation in this paper is both foundational and applied. It demonstrates how to create elementary conceptualizations based upon phenomenology and how to apply them to the case of thermoelectric devices. No microscopic interpretation or formalism is intended and no particular material is discussed. The result is a simple and visual representation of processes that can be used to suggest mathematically formal versions of spatially continuous processes.

In Section "Macroscopic dynamical processes," a short outline of the ideas behind continuum physics and the approach to the creation of spatially uniform dynamical models in thermal processes is given. Section "Phenomenology of thermoelectric processes" is devoted to the phenomenology of thermoelectric effects and demonstrates how to construct simple global representations of the underlying processes, leading to the simplest dynamical model of a Peltier device. A first discussion of the role of energy in these processes already leads to a proof of the equality of Peltier and 
Seebeck coefficients - suggesting that laborious microscopic arguments for the equality of Onsager's reciprocity relations are unnecessary.

These results suggest directly and simply how to write the transport equations in a thermoelectric material in spatially continuous form (Section "Continuous models of transport processes"). There we consider the coupled transport of substance, charge, and entropy, and the independent phenomenon of the diffusion of entropy. In sum, this leads to the well-known equations of thermoelectric materials without the need of much of the apparatus of irreversible thermodynamics.

Finally, in Section "Efficiency and figure of merit," we derive global Second Law efficiencies of thermoelectric devices for generators and heat pumps. The simple form found for generators lends itself to introduce and motivate the figure of merit $z T$ as a dimensionless combination of internal electric resistance, entropy conductance, and Seebeck coefficient.

\section{Macroscopic dynamical processes}

As mentioned in the Introduction, a macroscopic approach will be used to build the foundations for an understanding of thermoelectric processes and devices. This approach relies upon continuum physics and a direct entropic (caloric) representation of thermal processes. In this section, a brief outline of how to create models of dynamical thermal systems will be presented.

\section{Continuum physics}

Continuum physics (Truesdell and Toupin 1960; Truesdell and Noll 1965; Eringen 1971-1976) leads to a unified approach to macroscopic processes that use the same few basic steps for conceptualization and formulation of different types of phenomena. First, we have to agree on which physical quantities we are going to use as the fundamental or primitive ones. Other quantities are defined on their basis, and with their help laws are expressed. Second, there are the fundamental laws of balance of the quantities which are exchanged in processes, such as momentum, charge, amount of substance, or entropy; we call these quantities fluidlike. Third, we need particular laws governing the behavior of, or distinguishing between, different bodies; these laws are called constitutive relations. Last but not least, we need a means of relating different types of physical phenomena. The tool which permits us to do this is energy. We use the energy principle, that is, the law which expresses our belief that there is a conserved quantity which appears in all phenomena, and which has a particular relationship with each of the types of processes. A modern introduction to continuum thermodynamics that builds upon this approach and starts from the construction of spatially uniform models has been presented in Fuchs (2010).

The most basic constitutive relations result from the metaphoric interpretation of the intensive quantities associated with processes - speed with momentum, electric potential with charge, or temperature with entropy (Fuchs 2013a). These quantities are levels - they are described as being high or low by virtue of the projection of the schema of verticality onto the polarity which is constructed by our perception. Intensity results from the containment of a fluidlike quantity in a system: Pressure goes up if more liquid or gas is put into a container. This we call a capacitive relation. Differences of intensities are conceptualized as driving forces of processes. So the electric potential difference serves as a driving force for the flow of charge through a conductor, and a chemical potential difference is visualized as the driving force for the diffusion of a substance through a material. Such relations we call resistive characteristics. See Section "Entropy and models of dynamical thermal processes" for an example of the construction of a dynamical thermal model.

The notion of levels and level differences as driving forces is instrumental also for understanding the role of energy in physical processes (Section "Energy in physical processes"). We simply relate the power of processes to driving forces and flows, as Carnot did. This is the starting point for the approach used in this paper.

\section{Entropy and models of dynamical thermal processes}

Even though the balance of entropy is used as a stepping stone in continuum thermodynamics (Müller 1985; Jou, Casas-Vazquez, and Lebon 1996), we are commonly left to learning about this concept in the roundabout way of the traditional approach of a static theory of heat. Heat is identified with energy (in some form) and this quantity is used as the starting point for conceptualizations. In traditional presentations, entropy appears as a derived quantity and is commonly assumed to acquire a more foundational status only in statistical physics.

A direct macroscopic approach has been lacking even though suggestions for turning Carnot's (1824) caloric into 
a concept for basing macroscopic entropy upon have appeared time and again in the literature (Callendar 1911; Job 1972; Falk 1985; Fuchs 1987; Mares et al. 2008). A complete dynamical theory of heat inspired by continuum thermodynamics has been worked out by Fuchs (1996, 2010). It takes entropy (caloric) as one of two fundamental thermal quantities (the other is hotness as a foundation of temperature as the thermal potential). In particular, spatially uniform models can be found there of dynamical thermal processes suitable for a first encounter with modern thermodynamics as a macroscopic theory. Prior assumptions of equilibrium are just as alien and unnecessary as they are in mechanics or electrodynamics (Truesdell 1984). Equilibrium may or may not be attained, depending upon the situation and the model (see Jou and Casas-Vasquez 1988, for a non-equilibrium model of inertia in the conduction of heat). Second law analysis and optimization are standard elements of this approach (Bejan 1996; Sieniutycz and de Vos 2000; Fuchs 2010, Chapter 9).

Here is a brief outline of the theory of uniform dynamical thermal processes by way of a simple example. The aim is to facilitate (dynamical) modeling of thermoelectric generators and coolers as efficiently as possible (Section "Phenomenology of thermoelectric processes") and to prepare for continuous models at the same time (Section "Continuous models of transport processes"). Imagine a closed container with hot water cooling by emitting entropy to the environment. Entropy $(S)$ makes the water hot and because of the temperature difference between water and environment $\left(T-T_{a}\right)$, entropy will flow out $\left(I_{S}\right)$. The model consists of the dynamical version of the balance of entropy for the water (eq. [1]) and constitutive relations between entropy content and temperature (leading to the concept of entropy capacitance $K$ ) and between an entropy current and a temperature difference (leading to the concept of entropy conductance $G_{S}$ of a series of heat transfer layers). If we accept linear relations and spatially uniform conditions, the model takes the simple form:

$$
\begin{gathered}
\frac{d S}{d t}=I_{S}, \quad S(0)=S_{0} \\
S=K T \\
I_{S}=-G_{S}\left(T-T_{a}\right)
\end{gathered}
$$

The model can be transformed into an initial value problem for the temperature of the water as a function of time. It can be improved upon in various ways, for instance by making the constitutive quantities $K$ and $G_{S}$ temperature dependent or by using a lumped parameter approach in the case of spatially variable systems.

In uniform models, dissipation as a result of heat transfer takes place in thermal resistors between uniform elements, in this case, between the water and the environment. The rate of production of entropy, $\Pi_{S}$, equals $\Pi_{S}=\left(T_{1}-T_{2}\right) I_{S} / T_{2}$ (indices 1 and 2 denote positions in the direction of flow of entropy; see Section "Energy in physical processes”). In our example, this equals

$$
\Pi_{S}=G_{S} \frac{\left(T-T_{a}\right)^{2}}{T_{a}}
$$

The dynamical model for a Peltier device presented below in Section "Interpretation: a uniform dynamical model" contains elements of what we have used in this simple example.

\section{Energy in physical processes}

The treatment of energy in physical processes follows the example of Sadi Carnot (1824) and makes use of a proposal by Falk, Herrmann, and Schmid (1983). The former introduces the notion of power $\mathcal{P}$ as the result of a fluidlike quantity (volume of fluid, charge, entropy, amount of substance, momentum, or angular momentum) falling (or being pumped) through a potential difference (Fuchs 2010, Chapter 2); this corresponds to the notion of available power $\mathcal{P}_{a v}$ of a process. The latter was inspired by the Gibbs Fundamental Form of uniform systems and introduces us to the concept of energy carrier and associated energy flow: an energy current $I_{E}$ equals the current $I_{X}$ of a fluidlike quantity $X$ multiplied by the associated potential $\varphi_{X}$ at which it flows (Falk, Herrmann, and Schmid 1983; Fuchs 2010, Chapters 2-4). Together with the generalized balance of energy $E$ (assuming no production or destruction of energy), we obtain a complete picture of the role of energy in physical (and chemical) processes:

$$
\begin{gathered}
\mathcal{P}_{X}=\left(\varphi_{X 2}-\varphi_{X 1}\right)\left|I_{X}\right| \\
I_{E, X}=\varphi_{X} I_{X} \\
\frac{d E}{d t}=\sum I_{E, i}
\end{gathered}
$$

In the case of thermal processes, this leads to three relations well-known from irreversible thermodynamics or continuum thermodynamics:

$$
\mathcal{P}_{\text {th }}=\left(T_{2}-T_{1}\right)\left|I_{S}\right|
$$




$$
\begin{gathered}
I_{E, \text { th }}=T I_{S} \\
\mathcal{P}_{\text {diss }}=T I I_{S}
\end{gathered}
$$

$\mathcal{P}_{t h}$ is the thermal power (the rate at which energy is made available or used as a consequence of the flow of a current of entropy through a temperature difference). $\mathcal{P}_{\text {diss }}$ is the rate of dissipation of energy taking place at temperature $T$; this is equal to the energy used to produce entropy in an irreversible process. Here, all the equations have been written for simple uniform dynamical models; equivalent forms for spatially continuous forms will be given in Section "Continuous models of transport processes."

The approach is simple and rests upon a conceptualization that can make use of visual metaphors (Fuchs 2010, 2013b). Doing so introduces process diagrams that depict processes in terms of the flow, storage, and production of the fluidlike quantities, associated potentials and potential differences, and related energy quantities (available power, energy currents, and stored energy; Figure 1). The basic image of a process derives from Carnot's suggestion of using a waterfall as the archetypal process applicable to all types of phenomena (fluid, electric, thermal, chemical, mechanical). Clearly, this leads to a strong form of analogical reasoning in physics and physical chemistry.

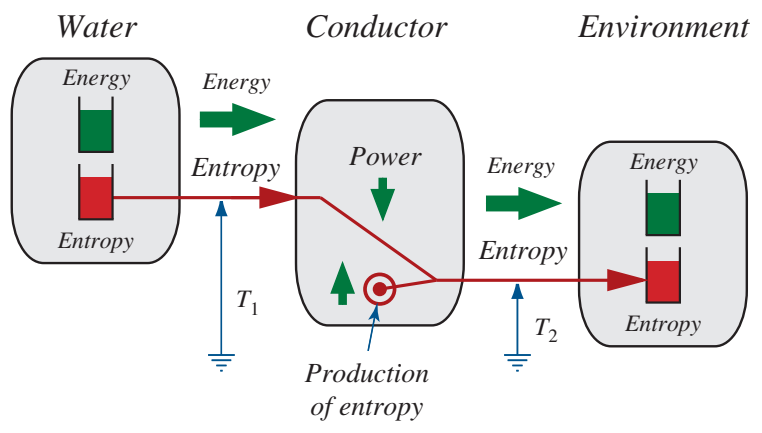

Figure 1 A process diagram of hot water cooling. Entropy flows downhill from the hot water into the cool environment (note the waterfall metaphor in the center). Entropy is stored in the water and the environment and produced in the heat transfer layers between the storage elements. When entropy falls, energy is released (made available) at a certain rate (power). When entropy flows into or out of an element, energy flows along with it

A process diagram for the example of cooling of water presented above can be seen in Figure 1. As discussed above, the body of water is treated as a uniform element, and entropy production takes place in the heat transfer layers treated as a thermal resistor placed between water and environment.

\section{Phenomenology of thermoelectric processes}

In this section, we will discuss an approach to creating a dynamical model of a Peltier device as a beginners' guide to thermoelectricity. The approach rests upon direct observation of some basic phenomena and interpretation in terms of the figures of mind presented in Section "Macroscopic dynamical processes." To demonstrate the power of the simple models, comparison of simulations with data taken in the laboratory can be performed.

\section{Observations}

Several versions of a simple experiment suitable for an introductory laboratory course are performed. A Peltier device (Melcor CP2-127-06L) is sandwiched between two copper plates (having the same surface area as the device) whose temperatures can be measured; the copper plates are insulated from the environment. The temperatures of the copper plates $\left(T_{1 C}\right.$ and $T_{2 C}$ in Figure $2 \mathrm{~A}$ ) serve as estimates of the surface temperatures of the Peltier device ( $T_{1}$ and $T_{2}$, respectively). The thermoelectric element is operated with a power supply whose voltage can be changed to different settings $\left(U_{S}\right)$ and is turned on and off during the experiment. Power supply, device, and an external resistive element form a simple circuit, and voltages $\left(U_{S}, U_{P D}, U_{\text {ext }}\right.$; the index $P D$ denotes the Peltier device) are recorded (Figure $2 \mathrm{~B})$.

Figure 2 shows a typical run of an experiment. The power supply is set to a fixed voltage for $100 \mathrm{~s}$; then it is turned off. In this experiment, the circuit is left open after the power supply was turned off. $U_{P D}$ then equals the open circuit voltage of the Peltier device after the power supply has been switched off.

Some conclusions can already be drawn by direct inspection of the results of measurements. The device works as an entropy pump driven by electricity (0-100 $\mathrm{s}$, Figure $2 \mathrm{~A}$ ); when the power supply is switched off and the temperatures of the faces of the device are different, an open circuit voltage is set up: the device works as a generator (100-300 s, Figure 2B); at the very beginning, the voltages across the device $\left(U_{P D}\right)$ and the external resistor $\left(U_{\text {ext }}\right)$ are almost equal, suggesting that the device has an internal resistance roughly equal to that of the external resistor $(1.0 \Omega)$; the fact that $U_{P D}$ and $U_{\text {ext }}$ become unequal when a temperature difference across the device is established (and grows with this difference) indicates that a "generator voltage" (thermoelectric 

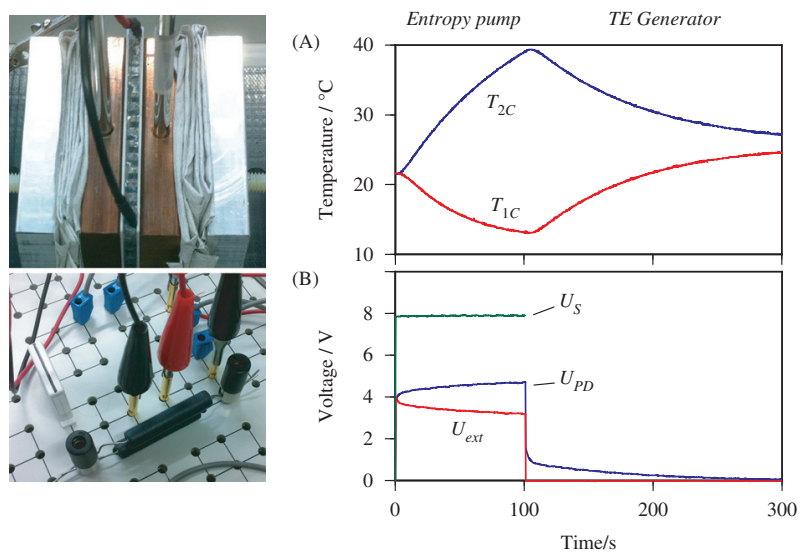

Figure 2 A Melcor CP2-127-06L device sandwiched between two copper plates (upper photograph) whose temperatures have been recorded (A). The device is in a circuit with a power supply set to $7.9 \mathrm{~V}$ (switched off after $100 \mathrm{~s}$ ) and an external resistor having $1.0 \Omega$ resistance (lower photograph, and data in B). Voltages across power supply $\left(U_{S}\right)$, Peltier device $\left(U_{P D}\right)$, and external resistor $\left(U_{\text {ext }}\right)$ are shown

voltage) is already present during heat pumping mode: the Peltier device seems to operate like a battery with internal resistance.

If we use different fixed voltages (and therefore different electric currents going through the device), the initial rate at which $T_{1}$ and $T_{2}$ spread (Figure $3 \mathrm{~A}$ ) is proportional to the electric current (Figure 3B). Since the rate of change of temperatures of the copper plates indicates the rate at which they loose or gain entropy, we can hypothesize that charge sweeps entropy along with it in proportion to the magnitude of its flow. Furthermore, we conclude from the fact that the first plate cools more slowly than the second heats up that entropy is produced by the Peltier device, most likely as a result of the conduction of charge through the device.
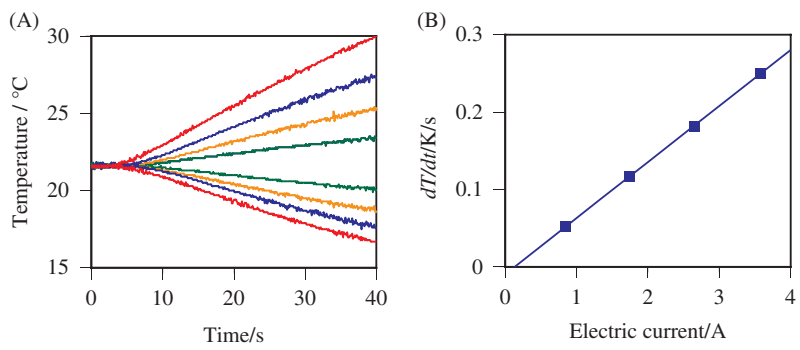

Figure 3 The Peltier device (upper photograph in Figure 2) is repeatedly operated in entropy pump mode with different values of the electric current. (A) Initial change of temperatures of copper plates touching the hot and cold sides of the Peltier device for four different currents. (B) Rate of change of temperature at hot side as a function of the electric current
The second part of the data shown in Figure 2, after the power supply has been switched off, can be used to investigate the generator function of the device. If we plot the open circuit voltage $\left(U_{P D}\right)$ as a function of temperature difference $\left(T_{2 C}-T_{1 C}\right)$, we see that the former is proportional to the latter (Figure 4A): a temperature difference across a Peltier device leads to a voltage and the device could be used as a generator.

We can change the experiment slightly by leaving the electric circuit closed after switching off the power supply. We notice that $U_{P D}$ is lower than before, and the smaller the resistance of the external resistor, the lower it will be. This allows us to produce a characteristic diagram of the device (Figure 4B): the electric current going through the device operating as a generator is a falling linear function of the voltage $U_{P D}$ across the device. Since this looks very much like the characteristic diagram of a battery modeled as an electrochemical generator having (constant) internal resistance, we suggest that a Peltier device is a thermoelectric generator having constant internal resistance.
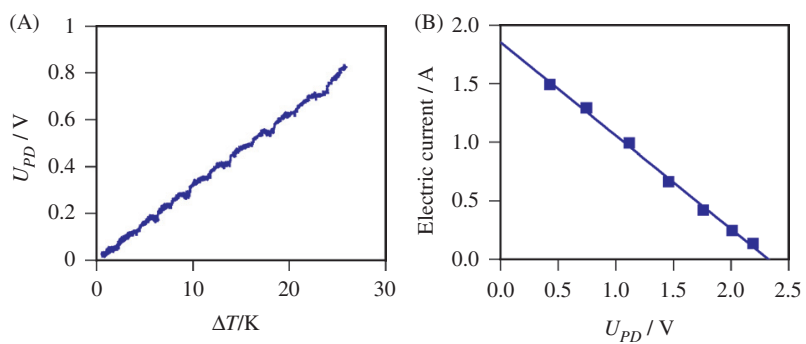

Figure 4 (A) Open circuit voltage of a Melcor CP2-127-06L Peltier element between two copper plates whose temperatures have been measured. (B) Terminal voltage if there is an electric current through the device. (This latter set of data is from the Peltier device between two bodies of water at different temperatures)

\section{Interpretation: a uniform dynamical model}

The interpretation of the behavior of the Peltier device rests upon a general observation and the concrete results of the experiments described in Section "Observations". This general observation is that the device, when operating, has a hot side and a cold side, and an electrically high side and a low side, respectively. Most simply, these observations mean that we are dealing with (coupled) thermal and electric processes: entropy and charge flow and are stored, and entropy is produced. Neglecting the coupling for the moment, the simplest model of the device is one consisting of two storage elements for entropy (to represent the hot and cold sides, respectively) 
and two electric capacitive elements (to represent two different potentials). Charge is allowed to flow (diffuse) between the capacitors, and entropy diffuses from the hotter to the colder thermal capacitor (Figure 5; see also Fuchs 2010, chapter 4). Both transport processes are known to be dissipative, that is, entropy is produced.

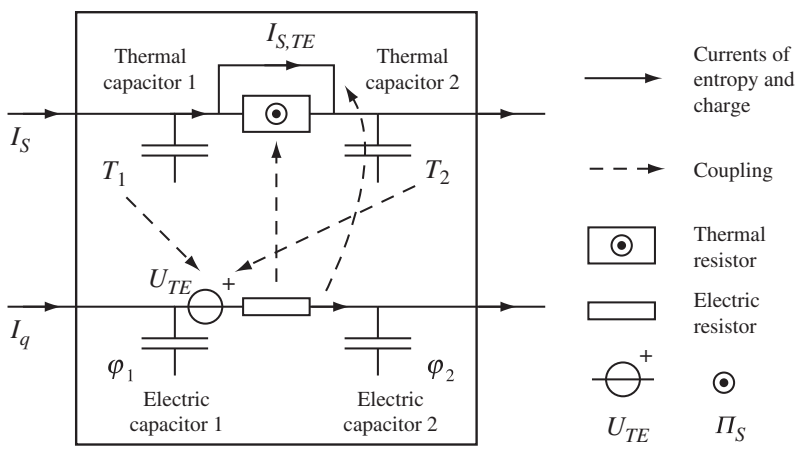

Figure 5 Simplest possible lumped parameter (uniform) dynamical model of a Peltier device. Thermally and electrically high and low faces are represented by capacitive elements. Transports of entropy $\left(I_{S}, I_{S, T E}\right)$ and of charge $\left(I_{q}\right)$ take place between these capacitors. Entropy flows conductively, is carried by charge, and is produced ( $\Pi_{S}$; source symbol at top center). A temperature difference sets up a thermoelectric voltage $U_{T E}$ ("electromotoric force")

We are most interested in the apparent coupling of the transports of charge and entropy. Overall, the experiments suggest that there are two types of coupling between electric and thermal processes. The result in Figure $3 \mathrm{~B}$ can be interpreted as follows. At $\Delta T=0$, there is a flow of entropy from one side of the device to the other that leads to the cooling of the first and the heating of the second. The rate of change of the temperatures is proportional to the entropy current (eqs [1] and [2]), and since the former is proportional to the electric current forced through the device, we may hypothesize that

$$
I_{S, T E}=\alpha I_{q}, \quad \text { if } T_{2}=T_{1}
$$

The index $T E$ stands for thermoelectric. Figuratively speaking, entropy is swept along with the electric charge flowing through the thermoelectric device. (This point should be clarified. The direction of flow of charge and entropy need not be the same; it depends upon the conductor. For example, in a p-type semiconductor, charge and entropy flow in the same direction, in an $n$-type conductor they flow in opposite directions. Thus it is possible to build a TE-device from (multiple) thermocouples using different materials. The device typically has a geometry as shown in Feldhoff and Geppert $(2014,70)$ or in Fuchs $(2010,175)$.) In Section "Continuous models of transport processes," we will interpret this as a phenomenon akin to convection where a fluid (here electric charge) carries a certain amount of entropy "stored" in it.

We call $\alpha$ the Peltier coefficient of the device. In general, with a temperature difference across the device, the current of entropy must be the result of two effects: the thermoelectric effect of entropy carried by charge $\left(I_{S, T E}\right.$, eq. [11]) and diffusion (conduction) of entropy $\left(I_{S(c)}\right.$, Fourier effect):

$$
I_{S}=\alpha I_{q}-G_{S}\left(T_{2}-T_{1}\right)
$$

On the other hand, the results shown in Figure 4A tell us that - for open circuit conditions - a voltage $U_{P D}=U_{T E}$ is set up across the device that is proportional to the temperature difference $\left(T_{2}-T_{1}\right)$ :

$$
U_{T E}=\varepsilon\left(T_{2}-T_{1}\right) \quad, \quad \text { if } I_{q}=0
$$

(Remember that voltages are negative potential differences.) $\varepsilon$ is the Seebeck coefficient of the thermoelectric device and $U_{T E}$ is the thermoelectric generator voltage analogous to the open circuit voltage (stemming from the "electromotoric force" or emf) of a battery. As we know, the Seebeck coefficient must be equal to the Peltier coefficient, $\varepsilon=\alpha$. Below, we will be able to give a rather simple argument for this claim based upon energy considerations (Sections "Energy in thermoelectric processes" and "Power and the equality of Seebeck and Peltier coefficients").

Finally, Figure 4B suggests that the thermoelectric device functions analogously to a battery with internal resistance $R$. Therefore, we hypothesize that, in general, the electric current through the device must be given by

$$
I_{q}=G\left(U_{P D}-\varepsilon\left(T_{2}-T_{1}\right)\right)
$$

$G=1 / R$ denotes the electric conductance. For a generator, going in the direction of the flow of charge, $\varepsilon\left(T_{2}-T_{1}\right)$ is negative, and so is $U_{P D}$; their difference will be positive, leading to a current from position 1 to position 2 in Figure 5.

The model is completed by adding the effects of entropy production. Only two processes are dissipative: diffusion of charge and diffusion of entropy. The former leads to

$$
\Pi_{S, e l}=\frac{1}{T_{2}} \frac{1}{G} I_{q}^{2}
$$

Entropy produced as a result of the conduction of entropy equals

$$
\Pi_{S, \text { th }}=\frac{1}{T_{2}} G_{S}\left(T_{2}-T_{1}\right)^{2}
$$


These equations are the results of eqs [5], [8] and [10] applied to our element. Electric power equals $R I_{q}^{2}$, and the power of the conductive (diffusive) current of entropy equals $G_{S}\left(T_{2}-T_{1}\right)^{2}$. For a finite body having a range of temperatures from $T_{1}$ to $T_{2}$, dissipation takes place at $T_{2}$ which means we divide the power of the dissipative process by this temperature (the result is the same for the model in Figure 5 running in either generator or heat pump mode).

The equations developed here can be combined with expressions for the thermal and electric capacitive elements to complete a dynamical model according to the ideas sketched in Figure 5. For higher accuracy, a device can be divided into a few elements in a single spatial dimension. Models of this type can be used to quantify the parameters of a thermoelectric device by comparing simulation results to data. Despite their simplicity, they work well. (A note on numerical methods: If the electric capacitance is given suitably small values, the initial value problem becomes stiff. Tools for solving initial value problems (IVPs) usually include methods for stiff differential equations that work very well on the present systems of IVPs. As an alternative, one may treat the electric process as running in (pseudo) steady state in which case storage of charge is not considered and the model becomes simpler, also for numerical methods.)

\section{Energy in thermoelectric processes}

Details of the role of energy vary slightly if a thermoelectric device is operated in generator or in pumping mode. For this reason, let us concentrate on the first of these cases in our discussion of power, energy transfer, and dissipation.

A process diagram of a thermoelectric generator looks like the following (Figure 6; the equivalent diagram for a device in heat pump mode is found in Fuchs 2010, 174). Overall, entropy enters the device at high temperature and leaves at low temperature. Charge is pumped through the device. The entropy leaving equals the entropy entering plus what is produced inside. Energy enters with entropy and leaves with entropy and charge.

In order to understand the operation of the device, we need to consider the power of various processes. Entropy flows downhill from $T_{1}$ to $T_{2}$ by two processes: by conduction $\left(I_{S(c)}\right)$ and coupled to charge $\left(I_{S, T E}\right)$; each of these flows releases energy at a certain rate. The energy made available by conduction $\left(\mathcal{P}_{t h(c)}\right)$ is dissipated:

$$
\mathcal{P}_{\text {diss }, t h}=-\left(T_{2}-T_{1}\right)\left|I_{S(c)}\right|
$$

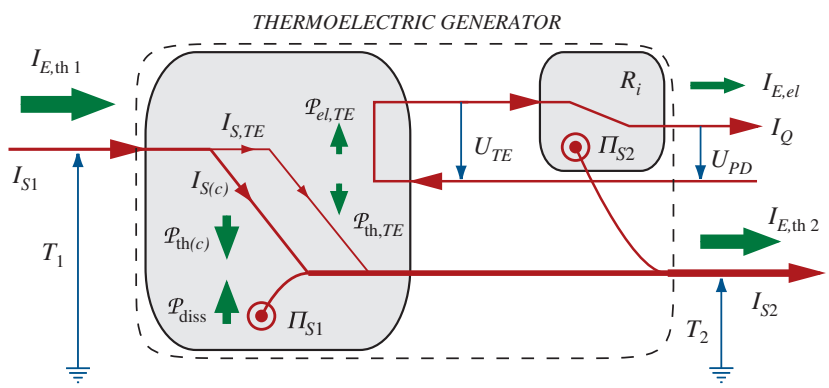

Figure 6 Steady-state process diagram of a thermoelectric device operated in generator mode. The electric resistive element is shown separately. Energy is made available in the fall of entropy $\left(I_{S 1}=I_{S(c)}+I_{S, T E}\right.$ where $I_{S(c)}$ is the conductive part of the current) from $T_{1}$ to $T_{2}\left(\mathcal{P}_{t h(c)}\right.$ and $\mathcal{P}_{t h, T E}$, respectively) and used partly to pump electric charge $\left(\mathcal{P}_{\text {el,TE}}\right)$. Entropy is produced as a result of the diffusion of entropy and charge $\left(\Pi_{S 1}\right.$ associated with $I_{S(c)}$ and $\Pi_{S 1}$ associated with $I_{q}$ )

The energy made available by the fall of entropy coupled to the flow of charge $\left(\mathcal{P}_{\text {th } T E}\right)$ is used for pumping charge: $\mathcal{P}_{\text {th }, T E}=-\mathcal{P}_{\text {el,TE}}$. This is the part of the thermal power that is initially non-dissipative:

$$
\mathcal{P}_{\text {th }, T E}=\left(T_{2}-T_{1}\right)\left|I_{S, T E}\right|
$$

Now we can create a simple argument based upon relations of power in the device that will lead to a proof of the equality of Seebeck and Peltier coefficients. Since $\mathcal{P}_{\text {th,TE }}=-\mathcal{P}_{\text {el,TE}}$, and after inserting the expressions from eqs [11] and [13], we obtain $\left(T_{2}-T_{1}\right) \alpha I_{q}=\varepsilon\left(T_{2}-T_{1}\right) I_{q}$, which means that

$$
\alpha=\varepsilon
$$

A slightly more sophisticated version of this argument and proof will be delivered in Section "Continuous models of transport processes" and efficiencies and the figure of merit will be introduced in Section "Efficiency and figure of merit."

\section{Continuous models of transport processes}

Spatially continuous models (dynamical or steady state) follow very directly from the ideas worked out in Section "Phenomenology of thermoelectric processes"; it is not difficult to convert eqs [12] and [14]. However, it may be useful to discuss the continuous case more carefully and at the same time, widen the scope by introducing the chemical nature of the transport processes in parallel with electric and thermal aspects. We will be rewarded 
with an enhanced visual conceptualization of thermoelectric processes and an introduction to the notions of coupled potentials, gradients of potentials, and entropy associated with amount of substance and charge of the (mobile) charge carriers (holes or electrons) in the material of the thermoelectric device.

\section{Transports of substance, charge, and entropy}

Consider a long, thin slab of a thermoelectric material (having a cross section $A$ and length $L$ ) that allows for quantities such as entropy or charge to flow in a single direction only (Figure 7). Densities and potentials of the quantities involved change in the direction of flow.

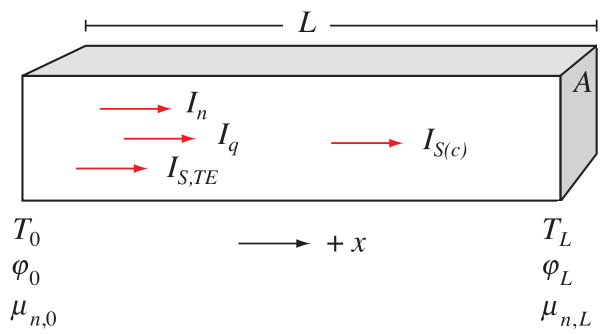

Figure 7 A slab of thermoelectric material (length $L$ and cross section $A$ ) allowing for transport of amount of substance $\left(I_{n}\right)$, charge $\left(I_{q}\right)$, and entropy $\left(I_{S}\right)$ in a single spatial dimension. Potentials shown are temperature $T$, electric potential $\varphi$, and chemical potential $\mu_{n}$. Transports of amount of substance, charge, and entropy are coupled. In addition, there is a conductive flow of entropy $\left(I_{S(c)}\right)$

The question now is how to create a complete description of processes with the least number of basic assumptions that lend themselves to graphic visualization. We know that entropy and charge must flow to create the phenomena of thermoelectricity. The transport of charge may be interpreted as a consequence of the diffusion of a charged substance (holes or electrons); if we give this substance not only charge but entropy as well, and if we allow entropy to diffuse independently, we can explain what we have discussed so far. In summary, all we need is a picture of the transport of amount of substance $n$ and of entropy $S$ through the material. To explain their flows, we need to consider the gradients of their potentials, chemical potential $\mu_{n}$, and temperature $T$, respectively.

\section{Combined potentials}

To describe the flow of amount of substance, we need the idea of chemical potential whose difference is the drive for currents of substance. However, if the substance has additional - non-chemical - properties such as charge or entropy, the gradients of electric potential and of temperature "pull" at the substance in parallel with the gradient of chemical potential.

To formalize this idea, we need to create the concept of combined potentials of a substance being electrically charged and charged with entropy. We use the image of an energy current being carried by a bundle of carriers, here amount of substance, charge, and entropy (see Falk, Herrmann, and Schmid 1983):

$$
I_{E}=\mu_{n} I_{n}+\varphi I_{q}+T I_{S}
$$

Here, $I_{S}$ is the total transport of entropy composed of the usual conductive part and the thermoelectric part associated with the transport of substance (which we may call a form of convection): $I_{S}=I_{S \text {, cond }}+I_{S, T E}$.

Charge is coupled to amount of substance, and so is a part of the entropy of the substance. If amount of substance flows, so do charge and entropy:

$$
\begin{gathered}
I_{q}=q_{n} I_{n} \\
I_{S, T E}=S_{n} I_{n}
\end{gathered}
$$

$q_{n}$ is the molar charge (charge per amount of substance); note that $q_{n}=z \mathcal{F}$ where $z= \pm 1$ and $\mathcal{F}$ is the Faraday constant. Analogously, $S_{n}$ is the entropy transported by the diffusing substance, per amount of substance (see Goupil et al. 2011, 1490; it is a kind of molar entropy, i.e., a constitutive quantity of the thermoelectric material). In other words, we imagine the substance in question to "hold" charge and entropy in accordance with these molar values, and when the substance flows, so do the associated amounts of charge and entropy. If we insert eqs [21] and [22] into eq. [20], we obtain

$$
I_{E}=\left(\mu_{n}+q_{n} \varphi+S_{n} T\right) I_{n}+T I_{S, \text { cond }}
$$

The quantity in parentheses is called thermo-electrochemical potential:

$$
\mu_{n, T E C}=\mu+q_{n} \varphi+S_{n} T
$$

In other words, we make this combined thermo-electrochemical potential responsible for the "pull" exerted upon the substance.

\section{Transport of substance}

We now assume that the first flow, substance together with its charge and entropy, is forced by the (negative) gradient of this combined potential. If we write this 
diffusive transport in terms of the current density of amount of substance driven by a gradient of the thermo-electro-chemical potential,

$$
j_{n}=-\sigma_{n} \frac{d}{d x} \mu_{n, \mathrm{TEC}}
$$

we have

$$
j_{n}=-\sigma_{n} \frac{d}{d x}\left(\mu_{n}+q_{n} \varphi+S_{n} T\right)
$$

$\sigma_{n}$ is the (chemical) conductivity of the substance diffusing through the thermoelectric material. If desired, we can rewrite this equation in terms of the flow of electric charge:

$$
j_{q}=-\sigma \frac{d}{d x}\left(\frac{\mu_{n, E C}}{q_{n}}\right)-\sigma \frac{S_{n}}{q_{n}} \frac{d T}{d x} \quad, \quad \sigma=q_{n}^{2} \sigma_{n}
$$

$\sigma$ is the electric conductivity, and $\mu_{n, E C}$ equals the sum of the first two terms on the right-hand side of eq. [24] which we call the electro-chemical potential. Note that $S_{q}=S_{n} / q_{n}$ equals the entropy per charge of the thermoelectric material. Here and in the following, we will assume that material properties are constant; results will be easier to read and to interpret.

\section{Transport of entropy}

The second transport is quite simple to visualize. Entropy flows (1) because it is carried by the diffusing (charged) substance and (2) because of a gradient of temperature (it diffuses):

$$
j_{S}=S_{n} j_{n}-\kappa_{S} \frac{d T}{d x}
$$

Here, $\kappa_{S}$ is the entropy conductivity of the material. The second term on the right is the well-known Fourier expression written for the conduction of the basic thermal extensive quantity, entropy. The first term on the right can be transformed with the help of eq. [21]:

$$
j_{S}=\frac{S_{n}}{q_{n}} j_{q}-\kappa_{S} \frac{d T}{d x}
$$

\section{Comparison with phenomenology}

We can take eqs [27] and [29] as the spatially continuous equivalents of the expressions formulated in eqs [12] and [14]. If we compare them, we obtain relations between the phenomenological Peltier and Seebeck coefficients and the material parameter entropy per charge:

$$
\alpha=\frac{S_{n}}{q_{n}}:=S_{q}, \quad \varepsilon=\frac{S_{n}}{q_{n}}:=S_{q}
$$

Interestingly, the graphical interpretation of the (local) transport processes used to formulate eqs [27] and [29] already suffices to suggest that the Peltier and Seebeck coefficients are equal. We can now write our transport equations of a spatially continuous thermoelectric material as follows:

$$
\begin{gathered}
j_{q}=-\sigma \frac{d}{d x}\left(\frac{\mu_{n, E C}}{q_{n}}\right)-\sigma \varepsilon \frac{d T}{d x} \\
j_{S}=\alpha j_{q}-\kappa_{S} \frac{d T}{d x}
\end{gathered}
$$

These equations are often given in a form with $j_{q}$ from eq. (31) inserted in the first term on the right-hand side of eq. [32]. Written in matrix form, we have

$$
\left(\begin{array}{c}
j_{q} \\
j_{S}
\end{array}\right)=-\left(\begin{array}{cc}
\sigma & \sigma \varepsilon \\
\sigma \alpha & \sigma \alpha \varepsilon+\kappa_{S}
\end{array}\right)\left(\begin{array}{c}
\frac{d}{d x}\left(\frac{\mu_{n, E C}}{q_{n}}\right) \\
\frac{d T}{d x}
\end{array}\right)
$$

This corresponds to what we find in the literature on thermoelectricity; see for example, Feldhoff and Geppert (2014) or Goupil et al. (2011).

\section{Power and the equality of Seebeck and Peltier coefficients}

As a last step in this section, let us revisit the point of the equality of Seebeck and Peltier coefficients. We already have two arguments that this should be so, the first based upon the overall reversible power of a device and the second based upon the phenomenological interpretation of material properties and transport phenomena relating to a thermoelectric material. Now we will take a closer look at the question of energy relations in the case of the continuous model.

The local (volume) density of the power ( $\mathfrak{p}$ ) of the thermal process - as a consequence of the fall of entropy through the gradient of temperature - is expressed as follows:

$$
\mathfrak{p}_{t h}=j_{S} \frac{d T}{d x}
$$

whereas the local balance of power takes the form

$$
-\mathfrak{p}_{\text {th }}=\mathfrak{p}_{\text {diss }, \text { th }}+\mathfrak{p}_{\text {diss }, e l}+\mathfrak{p}_{\text {el }}
$$

In simple terms, this tells us that energy is made available by a single process and used by three others, two of 
them dissipative. The first term on the right represents the dissipation rate due to thermal conduction; the second equals electric dissipation; and the third is the electric power of the device. If we introduce eq. [32] into eq. [34], we obtain

$$
\begin{aligned}
\mathfrak{p}_{t h} & =j_{S} \frac{d T}{d x} \\
& =\left(\alpha j_{q}-\kappa_{S} \frac{d T}{d x}\right) \frac{d T}{d x} \\
& =\alpha j_{q} \frac{d T}{d x}-\kappa_{S}\left(\frac{d T}{d x}\right)^{2}
\end{aligned}
$$

If we replace the first of the $d T / d x$ - terms according to eq. [31], we have

$$
\mathfrak{p}_{t h}=\alpha j_{q}\left(-\frac{1}{\sigma \varepsilon} j_{q}-\frac{1}{\varepsilon} \frac{d}{d x}\left(\frac{\mu_{n, E C}}{q_{n}}\right)\right)-\kappa_{S}\left(\frac{d T}{d x}\right)^{2}
$$

and finally, by using eq. [35]:

$\mathfrak{p}_{\text {diss }, t h}+\mathfrak{p}_{\text {diss }, e l}+\mathfrak{p}_{\text {el }}=\kappa_{S}\left(\frac{d T}{d x}\right)^{2}+\frac{\alpha}{\sigma \varepsilon} j_{q}^{2}+\frac{\alpha}{\varepsilon} j_{q} \frac{d}{d x}\left(\frac{\mu_{n, E C}}{q_{n}}\right)$

The terms on the right have forms we recognize from our conceptualization of energy in physical processes (Section "Energy in physical processes"). The first is indeed equal to dissipation in thermal conduction (it is the local version of eq. [16]), the second takes the form of electric dissipation if $\alpha=\varepsilon$ (it is the local equivalent of eq. [15]), and if we again set $\alpha=\varepsilon$, the third is equal to the power of pumping of a current of charge $j_{q}$ through the gradient of the electro-chemical potential per molar charge (giving us the electric power of the device).

\section{Continuous dynamical and steady-state models}

Now that we know how to express the pertinent transport equations, we can formulate complete continuous models by introducing the constitutive relations into the relevant laws of balance. For charge and for entropy, these are

$$
\begin{aligned}
& \frac{\partial \rho_{q}}{\partial t}+\frac{\partial j_{q}}{\partial x}=0 \\
& \frac{\partial \rho_{S}}{\partial t}+\frac{\partial j_{S}}{\partial x}=\pi_{S}
\end{aligned}
$$

where $\rho$ stands for volumetric density and $\pi_{S}$ is the volumetric density of the production rate of entropy. These are the single-dimensional versions of the general laws of balance of charge and entropy known from continuum physics (Müller 1985; Fuchs 2010, Chapter 11). The local rate of production of entropy equals the local dissipation rate (which is the sum of thermal and electric contributions as in eq. [36]) divided by the local temperature:

$$
\pi_{S}=\frac{1}{T} \kappa_{S}\left(\frac{d T}{d x}\right)^{2}+\frac{1}{T} \frac{1}{\sigma} j_{q}^{2}
$$

\section{A steady-state model}

In steady state, the derivatives with respect to time vanish in eqs [37] and [38]. The former then tells us that the current density of charge must be (temporally and spatially) constant, and the latter turns into

$$
\frac{d}{d x}\left(\alpha j_{q}-\kappa_{S} \frac{d T}{d x}\right)=\frac{1}{T} \kappa_{S}\left(\frac{d T}{d x}\right)^{2}+\frac{1}{T} \frac{1}{\sigma} j_{q}^{2}
$$

(Note that the derivative of the first term on the left will be equal to zero.) To obtain global results similar to those suggested in Section "Interpretation: a uniform dynamical model" (see eqs [12] and [14]), we must solve the differential equation for $T(x)$. In general, when the material coefficients $\alpha, \sigma$, and $\kappa_{S}$ are arbitrary functions of $T$, we cannot find simple solutions. One commonly used result holds for constant $\alpha_{E}=T \alpha$, constant $\kappa_{E}=T \kappa_{S}$, and constant $\sigma$. In this case, the electric current is constant and the entropy currents at the ends of the slab in Figure 7 are equal to

$$
x=0: \quad I_{S}(x=0)=\frac{\alpha_{E}}{T_{0}} I_{q}-\frac{G_{E}}{T_{0}}\left(T_{L}-T_{0}\right)-\frac{1}{2} \frac{1}{T_{0}} R I_{q}^{2}
$$

$$
x=L: \quad I_{S}(x=L)=\frac{\alpha_{E}}{T_{L}} I_{q}-\frac{G_{E}}{T_{L}}\left(T_{L}-T_{0}\right)+\frac{1}{2} \frac{1}{T_{L}} R I_{q}^{2}
$$

Here, $G_{E}$ can be interpreted as the conductance for the energy current accompanying the transport of entropy $\left(G_{E}=T G_{S}\right)$. Note that these are rather special results that do not hold in general. Nevertheless, they will be useful in our discussion of efficiency in the next section.

\section{Efficiency and figure of merit}

In this section, global Second Law efficiencies will be derived for steady-state operation and a natural definition 
of the figure of merit of thermoelectric devices will be defined. The discussion will be based upon a form of an entropy current at $x=0$ suggested by eq. [41] and of the electric current by eq. [14]:

$$
\begin{gathered}
I_{q}=\frac{1}{R}\left(U_{P D}-\alpha \Delta T\right) \\
I_{S}=\alpha I_{q}-G_{S} \Delta T-\frac{1}{2} \frac{1}{T_{0}} R I_{q}^{2}
\end{gathered}
$$

In the following, $\alpha, G=1 / R$, and $G_{S}$ will be assumed to be constant. It turns out that the last term on the right side of eq. [44] is small compared to the others when the device is used in generator mode. It cannot be neglected, however, when we discuss the Second Law efficiency of a device used as a heat pump.

\section{Second Law and First Law efficiencies}

A natural measure of efficiency of heat engines - or any other type of device for that matter - can be gleaned from Figure 6 or the equivalent process diagram for a heat pump (see Fuchs 2010, chapter 4). This measure, called Second Law efficiency $\eta_{I I}$, is defined by the ratio of useful power to available power:

$$
\eta_{I I}=\frac{\mathcal{P}_{u s e}}{\mathcal{P}_{a v}}
$$

Note that $\eta_{I I}=1$ for a Carnot heat engine. The usual thermal efficiency (First Law efficiency $\eta_{I}$ ) is related to $\eta_{I I}$ as follows:

$$
\eta_{I}=\eta_{C} \eta_{I I}, \quad \eta_{C}=1-T_{2} / T_{1}
$$

Here, $\eta_{C}$ is the well-known Carnot factor.

\section{Efficiency of a thermoelectric generator}

Let us now discuss the Second Law efficiency of a thermoelectric device running in generator mode as in Figure 6. Energy is made available at a rate $\left(T_{1}-T_{2}\right) I_{S 1}$ and it is usefully employed (not dissipated) at a rate equal to $U_{P D} I_{q}$. Therefore, this natural measure of efficiency is defined by

$$
\eta_{I I}=\frac{U_{P D} I_{q}}{I_{S 1} \Delta T}
$$

Remember that, in this case, the third term on the right side of eq. [44] can be neglected. Furthermore, if the device is in a simple circuit having an external resistive element with resistance $R_{\mathrm{ext}}$, the electric current through the element is given by $I_{q}=-\varepsilon \Delta T /\left(R+R_{\mathrm{ext}}\right)$, and $\eta_{I I}$ becomes

$$
\eta_{I I}=\frac{R_{\mathrm{ext}}}{R+\left(R+R_{\mathrm{ext}}\right) \frac{R G_{s}}{\alpha^{2}}} \frac{R}{R+R_{\mathrm{ext}}}
$$

There is only a single external parameter, $R_{\mathrm{ext}}$, determining the efficiency. Beyond that, $\eta_{I I}$ depends upon the parameters of the device. Most interestingly, there is a figure of merit $z T$,

$$
z T=\frac{\alpha^{2}}{R G_{S}}=\frac{\alpha^{2} \sigma}{\kappa_{S}}
$$

so that $\eta_{I I} \rightarrow R_{\mathrm{ext}} /\left(R+R_{\mathrm{ext}}\right)$ for $z T \rightarrow \infty$. Efficiency as a function of $R_{\text {ext }}$ is shown for the Melcor CP2-127-06L device and two hypothetical devices with different $z T$ in Figure 8A. For given $z T$, the efficiency has a maximum at

$$
R_{\mathrm{ext}, \mathrm{opt}}=\sqrt{1+z T} R
$$

The meaning of $z T$ is quite clear. First, it is dimensionless and only dependent upon thermoelectric material or device properties. It grows with an increasing Seebeck or Peltier coefficient (which measures the strength of the thermoelectric effect) and increasing electric conductivity (which decreases electric dissipation) and decreasing entropy conductivity (which decreases the unwanted diffusion of entropy and related dissipation).

\section{Efficiency of a thermoelectric heat pump}

Let us now discuss the Second Law efficiency of a Peltier device used as a heat pump. In this case, the efficiency is defined by the ratio of (net) entropy pumping power and the electric power:

$$
\eta_{I I}=\frac{I_{S 1} \Delta T}{U_{P D} I_{q}}
$$

If we introduce eqs [43] and [44], we notice that two external parameters - such as the voltage applied to the device and the temperature difference across it - have to be specified for the efficiency to be defined:

$$
\eta_{I I}=\left[\frac{U_{P D}-\alpha \Delta T(1+1 / z T)}{U_{P D}-\alpha \Delta T} \alpha-\frac{1}{2} \frac{1}{T_{0}}\left(U_{P D}-\alpha \Delta T\right)\right] \frac{\Delta T}{U_{P D}}
$$

If the second term in the brackets could be neglected, and if $z T$ were to be made large, the efficiency would approach a value of $\alpha \Delta T / U_{P D}$. Numerical values of the Second Law efficiency of the Melcor CP2-127-06L device 

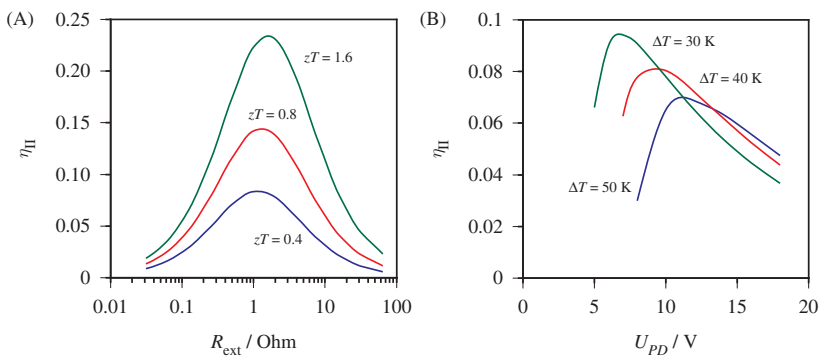

Figure 8 Results of simulations of the model developed here. (A) Second Law efficiency of a Peltier device used as a generator. The efficiency is shown as a function of the resistance of an external ohmic resistor for different figures of merit. The values assume $R=$ $1.0 \Omega$ (as for the Melcor CP2-127-06L device that has $z T=0.8$ ). (B) Second Law efficiency for the same Peltier device $(z T=0.8)$ operated as a heat pump as a function of driving voltage $U_{P D}$ across the element, for different temperature differences. The results shown here are those of a finite difference model

for different temperature differences are shown in Figure 8B.

\section{Conclusion}

It is indeed possible to create the background for an understanding of thermoelectric processes in a simple and straightforward manner. The conceptual tools for this approach are a combination of continuum thermodynamics and the forms of analogical reasoning afforded by it. Mechanics, electricity, chemistry, and heat can be based upon strictly analogous structures if momentum, charge, amount of substance, and entropy and their related potentials (velocity, electric and chemical potentials, and temperature) are used as primitive quantities. Macroscopic entropy can be visualized as the caloric of the caloric theory suitably extended by the requirement that caloric is produced in irreversible processes (Falk 1985; Fuchs 1996; Mares et al. 2008). In this form, thermodynamics joins the other macroscopic theories of physics and physical chemistry as an example of the conceptualization of forces of nature (Fuchs 2013a, 2013b).

As we have seen here, the most easily accessible theory of a thermoelectric device is a spatially uniform dynamical model suggested to us directly by the phenomenology of thermoelectric processes. This model serves as a first-order computational model and suggests how to write the spatially continuous transport relations. Identification of the power of various elementary processes or, even more simply, association of Peltier and
Seebeck coefficients with the (molar) entropy swept along by the diffusing (charged) substance, leads to a one-line proof of the equality of these coefficients. There is no need for laborious microscopic arguments for the equality of Onsager's reciprocity relation.

Finally, if we use the Second Law efficiency suggested by modern thermodynamics as a natural measure of efficiency, we can derive a simple result - at least for thermoelectric generators - that allows for a direct interpretation of the figure of merit composed of electric conductance, Seebeck coefficient, and entropy conductance.

\section{References}

Bejan, A. 1996. Entropy Generation Minimization. Boca Raton, FL: CRC Press.

Callendar, H. L. 1911. “The Caloric Theory of Heat and Carnot's Principle." Proceedings of the Physical Society (London) 23:153-89.

Carnot, S. 1824. Reflexions sur la puissance motrice du feu et sur les machines propres a developper cette puissance. Paris: Bachelier. Reflections on the Motive Power of Fire. Translated by R. H. Thurston, Dover Publications, New York (1960).

Eringen, A. C. 1971-1976. Continuum Physics, Vols. I-IV. New York: Academic Press.

Falk, G. 1985. "Entropy, a Resurrection of Caloric-A Look at the History of Thermodynamics." European Journal of Physics 6:108-15.

Falk, G., F. Herrmann, and G. B. Schmid. 1983. “Energy Forms or Energy Carriers?” American Journal of Physics 51:1074-7.

Feldhoff, A., and B. Geppert. 2014. “A High-Temperature Thermoelectric Generator Based on Oxides." Energy Harvesting and Systems 1(1-2):69-78.

Fuchs, H. U. 1987. "Entropy in the Teaching of Introductory Thermodynamics.” American Journal of Physics 55:215-19.

Fuchs, H. U. 1996. The Dynamics of Heat. New York: Springer.

Fuchs, H. U. 2010. The Dynamics of Heat. A Unified Approach to Thermodynamics and Heat Transfer, 2nd ed. Graduate Texts in Physics. New York: Springer.

Fuchs, H. U. 2013a. "From Image Schemas to Narrative Structures in Science. Symposium on Conceptual Metaphor and Embodied Cognition in Science Learning." Proceedings of the ESERA Conference 2013, Cyprus.

Fuchs, H. U. 2013b. "The Narrative Structure of Continuum Thermodynamics.” Proceedings of the ESERA Conference 2013, Cyprus.

Goupil, C., W. Seifert, K. Zabrocki, E. Müller, and G. F. Snyder. 2011. "Thermodynamics of Thermoelectric Phenomena and Applications.” Entropy 13:1481-517.

Job, G. 1972. Neudarstellung Der Wärmelehre: Die Entropie Als Wärme. Frankfurt: Akademische Verlagsgesellschaft.

Jou, D., and J. Casas-Vázqzez. 1988. “Extended Irreversible Thermodynamics of Heat Conduction." European Journal of Physics 9:329-33.

Jou, D., J. Casas-Vazquez, and G. Lebon. 1996. Extended Irreversible Thermodynamics, 2nd ed. Berlin: Springer. 
Mares, J. J., P. Hubik, J. Sestak, V. Spicka, J. Kristofik, and J. Stavek. 2008. "Phenomenological Approach to the Caloric Theory of Heat." Thermochimica Acta 474:16-24.

Müller, I. 1985. Thermodynamics. Boston, MA: Pitman.

Sieniutycz, S., and A. de Vos. 2000. Thermodynamics of Energy Conversion and Transport. Berlin: Springer.
Truesdell, C. A. 1984. Rational Thermodynamics. New York: Springer. Truesdell, C. A., and R. A. Toupin. 1960. "The Classical Field Theories." In Encyclopedia of Physics, vol III/1, edited by S. Flügge. Berlin: Springer-Verlag.

Truesdell, C. A., and W. Noll. 1965. "The Non-Linear Field Theories of Mechanics." In Encyclopedia of Physics, vol III/3, edited by S. Flügge. Berlin: Springer-Verlag. 